\title{
ISQUEMIA E REPERFUSÃO HEPÁTICA TOTAL ASSOCIADA AO ESTADO DE CHOQUE HEMORRÁGICO CONTROLADO - EFEITOS NO SEQÜESTRO DE NEUTRÓFILOS NO FÍGADO DO RATO
}

\author{
TOTAL HEPATIC WARM ISCHEMIAAND REPERFUSION ASSOCIATED WITH \\ CONTROLLED HEMORRHAGIC SHOCK: EFFECTS OF NEUTROPHIL \\ SEQUESTRATION IN RAT LIVER
}

\author{
Mario Mantovani ,TCBC-SP ${ }^{1}$ \\ Mauro José Fontelles, TCBC-SP ${ }^{2}$ \\ Élcio Shiyoiti Hirano ${ }^{3}$ \\ Rosana Celestina Morandin ${ }^{4}$ \\ André Almeida Schenka ${ }^{5}$
}

\begin{abstract}
RESUMO: Objetivo: O propósito deste trabalho experimental foi estudar os efeitos da isquemia e reperfusão hepática total sobre o acúmulo de neutrófilos no fígado de ratos, em condições de normalidade e submetidos ao estado de choque hemorrágico controlado. Método: Trinta e dois ratos Wistar, machos, foram divididos em quatro grupos de oito animais cada: grupo Controle, submetido à laparotomia com um período de 60 minutos de observação; grupo Choque, submetido a choque hemorrágico controlado (PAM = $40 \mathrm{mmHg}, 20 \mathrm{~min}$.) seguido de reposição volêmica (Ringer lactato + sangue, 3:1) e reperfusão (60 min.); grupo Pringle, submetido a isquemia hepática total (15 min.) e reperfusão (60 min.); grupo Total submetido a choque hemorrágico controlado $(15 \mathrm{~min}$.) seguido de reposição volêmica (Ringer lactato + sangue, $3: 1)$ mais isquemia hepática total (15 min.) e reperfusão (60 min.). A dosagem do lactato arterial e déficit de base foram utilizados para caracterizar o estado de choque hemorrágico com baixa perfusão tecidual. Após a morte dos animais, procedeu-se à contagem de neutrófilos no tecido hepático. Resultados: Na contagem de neutrófilos no fígado o grupo Pringle diferiu dos grupos Choque e Total, os quais

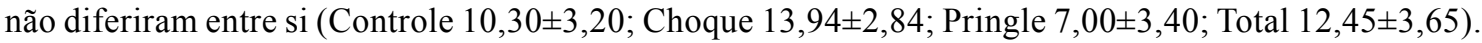
Conclusão: Em ratos submetidos a estado de choque hemorrágico controlado, associado à isquemia hepática total de 15 minutos, seguido de 60 minutos de reperfusão, não ocorreu acúmulo significativo de neutrófilos no fígado.
\end{abstract}

Descritores: Isquemia; Reperfusão; Choque Hemorrágico; Neutrófilos; Fígado; Ratos wistar; Hipovolemia.

1. Professor Titular e Chefe da Disciplina de Cirurgia do Trauma do Departamento de Cirurgia da Faculdade de Ciências Médicas UNICAMP; Coordenador do Laboratório de Investigação em Cirurgia do Trauma (LICIT) do Núcleo de Medicina e Cirurgia Experimental da FCM - UNICAMP.

2. Doutor em Cirurgia do Trauma; Cirurgião do Hospital do Pronto-Socorro Municipal de Belém; Professor da Disciplina de Anatomia Humana da Universidade da Amazônia - UNAMA.

3. Mestre em Cirurgia do Trauma; Médico do Hospital das Clínicas da Faculdade de Ciências Médicas - UNICAMP.

4. Doutora em Ciências Médicas - Anatomia Patológica; Bióloga do Laboratório de Investigação em Cirurgia do Trauma (LICIT) UNICAMP.

5. Médico Patologista da Faculdade de Ciências Médicas - UNICAMP.

Recebido em 06/02/2002

Aceito para publicação em 18/02/2003

Trabalho realizado no Laboratório de Investigação em Cirurgia do Trauma (LICIT) da Disciplina de Cirurgia da Faculdade de Ciências Médicas da UNICAMP - Campinas, S.P., no Núcleo de Medicina e Cirurgia Experimental da FCM - UNICAMP. 


\section{INTRODUÇÃO}

O fígado constitui o órgão mais atingido no trauma abdominal fechado, superado apenas pela lesão do baço. Nas lesões lobares extensas, associadas com graves desinserções vasculares envolvendo as veias supra-hepáticas, a mortalidade pode ocorrer em $50 \%$ dos $\operatorname{casos}^{1,2}$. Nestes pacientes, o clampeamento da tríade portal, conhecido como manobra de Pringle ${ }^{3}$, tem sido o procedimento padrão mais utilizado pelos cirurgiões para reduzir o risco de hemorragia maciça durante a abordagem cirúrgica do órgão. No entanto, esta manobra pode induzir à lesões hepáticas caracterizadas por alterações próprias da síndrome de isquemia e reperfusão $(\mathrm{I} / \mathrm{R})^{4,5}$.

Embora esta técnica comporte-se como uma maneira segura em reduzir o sangramento, muita controvérsia ainda existe sobre o tempo total de tolerância hepática à isquemia. Deste modo, estudos têm sido realizados para avaliar o tempo máximo em que o órgão pode suportar sem sofrer lesões e, também, para avaliar, de forma comparada, os efeitos da manobra quando executada de maneira intermitente, sendo o acúmulo de neutrófilos no tecido hepático um marcador confiável na quantificação destas lesões ${ }^{6,7}$.

O propósito deste trabalho experimental foi estudar os efeitos da isquemia e reperfusão hepática total, quantificados pelos níveis de acúmulo de neutrófilos no fígado de ratos em condições de normalidade e, também, submetidos ao estado de choque hemorrágico controlado.

\section{MÉTODO}

Todos os procedimentos seguiram a orientação do protocolo previamente estabelecido pela Disciplina de Cirurgia do Trauma e, aprovado pela Comissão de Ética na Experimentação Animal do Instituto de Biologia da UNICAMP.

Foram utilizados 32 ratos machos, adultos, da linhagem Wistar (CEMIB UNICAMP, Campinas, SP), com pesos que variaram de 185 a 250 gramas $(212,65 \pm 20,79)$ e com idade aproximada de oito semanas. Todos foram mantidos em condições ambientais controladas de luminosidade e temperatura, recebendo dieta padrão constituída de ração NUVILAB CR-1 (Nuvital Nutrientes Ltda., Paraná, Brasil) e água 'ad libitum'.

Os ratos foram aleatoriamente distribuídos em quatro grupos de oito animais cada:
- Grupo Controle - Ratos em condições de normalidade, submetidos, unicamente, à laparotomia e, posteriormente, a um período de 60 minutos de observação, equivalente ao período de reperfusão nos demais grupos;

- Grupo Choque - Ratos submetidos à sangria com instalação do estado de choque hemorrágico controlado, com pressão arterial média (PAM) mantida em $40 \mathrm{mmHg}$, por um período de 20 minutos, seguido da reposição volêmica com solução de Ringer lactato e sangue total (3:1) até atingir a PAM de $80 \mathrm{mmHg}$, com posterior período de reperfusão de 60 minutos.

- Grupo Pringle - Ratos submetidos à laparotomia seguida por 15 minutos de manobra de Pringle e, posterior período de reperfusão hepática total de 60 minutos.

- Grupo Total - Ratos submetidos à sangria com instalação do estado de choque hemorrágico controlado, com PAM mantida em $40 \mathrm{mmHg}$, por um período de 20 minutos, seguido da reposição volêmica com solução de Ringer Lactato e sangue total (3:1) até atingir a PAM de $80 \mathrm{mmHg}$. A seguir, foram submetidos à laparotomia e a 15 minutos de manobra de Pringle, seguida de reperfusão hepática total por um período de 60 minutos.

Os animais foram submetidos a jejum de 12 horas na noite anterior ao experimento. Os procedimentos cirúrgicos foram realizados sob anestesia intraperitoneal (IP) com uma solução de cloridrato de cetamina, $80 \mathrm{mg} / \mathrm{Kg}$ (Cristália, Itapira, SP) + cloridrato xilazina, $10 \mathrm{mg} / \mathrm{Kg}$ (Bayer S.A., São Paulo, SP) e, sulfato de atropina (Halex Istar, são Paulo, SP) $0,05 \mathrm{mg} / \mathrm{Kg}$. Oxigênio foi administrado num fluxo de 2 1/min., fornecido sob campânula plástica semi-aberta. Após indução anestésica, foi realizada depilação e os animais colocados em posição supina sobre prancha com aquecimento elétrico controlado.

Usando-se técnica anti-séptica, a artéria carótida e veia jugular, direitas, foram dissecadas e cateterizadas com cateteres de polietileno (PE 40), previamente heparinizados, que foram utilizados para efetivação da sangria com estabelecimento do estado de choque hemorrágico controlado, além da coleta de amostras de sangue para exames e infusão de líquidos para reposição volêmica, respectivamente. A seguir, a artéria femoral direita foi dissecada e cateterizada para controle da PAM dos animais (Registrador Mingograf 804. Siemens-Elema, Alemanha).

Durante todas as fases do experimento as seguintes variáveis foram monitorizadas continuamen- 
te: eletrocardiograma (ECG), PAM, freqüência cardíaca (FC), freqüência respiratória (FR) e temperatura retal (TR), a qual foi mantida entre $36^{\circ} \mathrm{C}-38^{\circ} \mathrm{C}$, controlada por aquecimento externo. Medidas das pressões parciais do gás carbônico $\left(\mathrm{pCO}_{2}\right)$ e do oxigênio $\left(\mathrm{pO}_{2}\right.$ ), dos eletrólitos (sódio, potássio), da reserva de base (BE), do lactato e $\mathrm{pH}$ sangüíneos, todos arteriais, foram obtidas após estabilização dos sinais vitais iniciais (SVI), no início da fase de reperfusão (RP0), aos 30 minutos de reperfusão (RP30) e aos 60 minutos de reperfusão (RP60).

As variáveis utilizadas para caracterizar o estado de choque hemorrágico controlado com baixa perfusão tecidual incluíram as medidas do $\mathrm{pH}$, da reserva de base e dosagem do lactato sérico nas fases estabelecidas pelo protocolo do experimento. O estado de choque hemorrágico controlado foi obtido pela sangria, a intervalos regulares, de volumes equivalentes a 5\% da volemia, até a obtenção dos níveis pressóricos iguais a $40 \mathrm{mmHg}$ de PAM para os animais do grupo Choque e Total (Figura 1). Retiradas posteriores foram efetuadas, se necessário. A laparotomia mediana foi realizada com hemostasia rigorosa dos vasos sangüíneos. O ligamento hepatoduodenal (artéria hepática, veia porta e ducto biliar) foi identificado e pinçado (manobra de Pringle) com clampe arterial delicado por 15 minutos. Após o período de reperfusão de 60 minutos os animais foram mortos por exsanguinação.
Fragmento dos lobos medianos dos fígados, previamente lavados em solução salina fisiológica, fixados em formalina a $10 \%$ e embebidos em parafina, foram submetidos a cortes histológicos de $4 \mathrm{~mm}$ de espessura e à coloração pela por hematoxilina/ eosina para estudo histológico e contagem do número de neutrófilos por campo. A leitura das lâminas foi realizada de maneira aleatória e o resultado final da contagem de neutrófilos, em cada corte, foi obtido pela média aritmética dos valores relativos a 10 campos microscópicos, com aumento de 400x, alternando-se dois campos sem leitura para cada campo considerado.

Nas comparações entre os grupos a significância estatística foi avaliada através da Análise de Variância (ANOVA de um critério - Teste F). Na presença de diferenças entre os grupos, estas foram identificadas pelo método de StudentNewman-Kuels. Adotou-se o nível de significância de $5 \%$ (p £ 0,05). Os valores de " $p$ " foram obtidos através do programa estatístico SAS - versão 6.12 (Statistical Analysis System Software - Cary, NC, USA).

\section{RESULTADOS}

Neste experimento, os grupos foram homogêneos, pois não ocorreram diferenças significativas quanto aos pesos dos animais estudados.

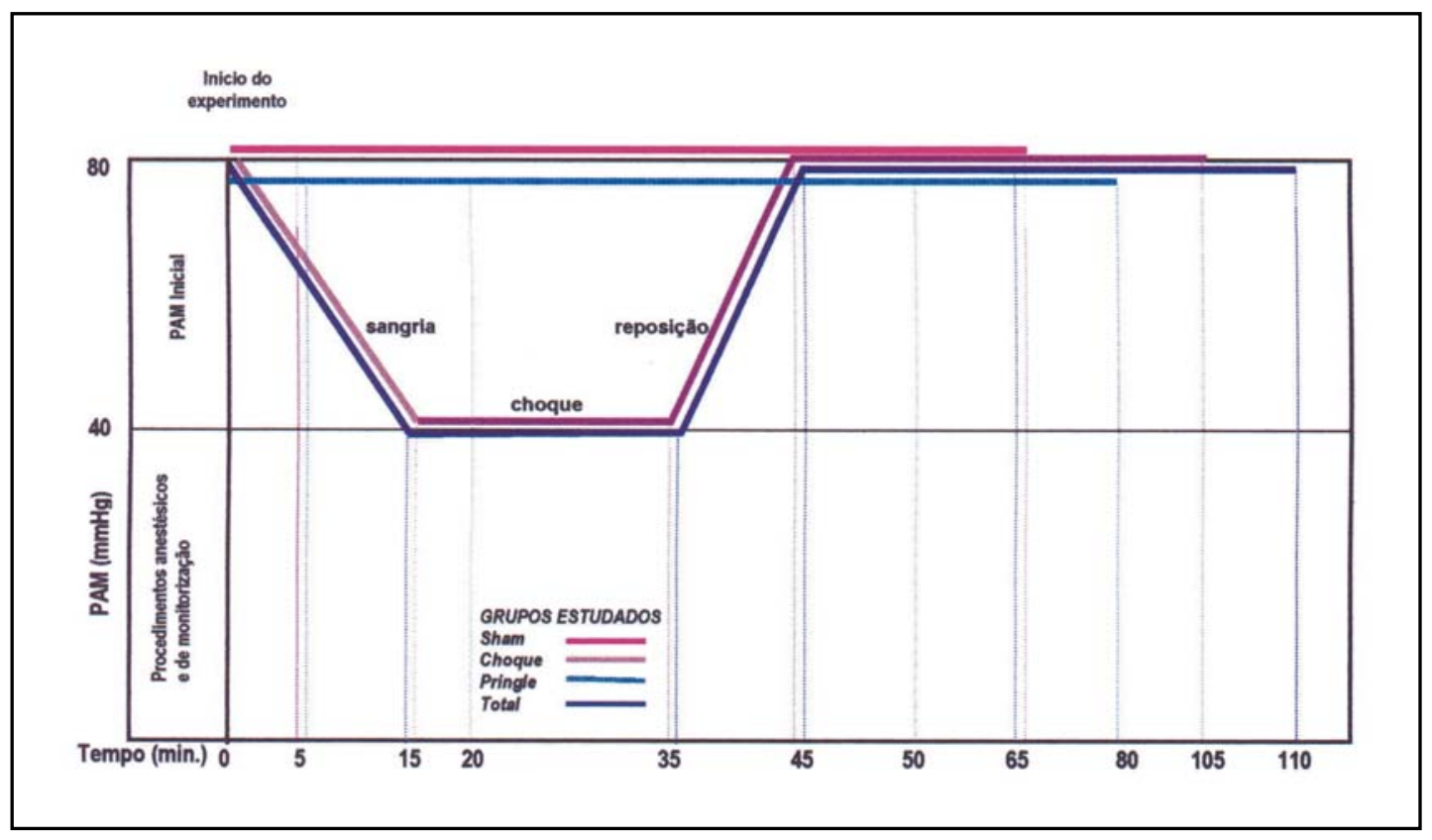

Figura 1 - Esquema de choque por hemorragia controlada. 
Em todos os animais as variáveis de interesse encontravam-se dentro dos limites de normalidade demonstrando que todos os animais foram retirados da mesma população. Os valores da média \pm desvio padrão, para cada grupo estudado, estão representados na tabela 1 .
Para cada grupo e, durante todas as fases de cada experimento, as medidas da PAM, FR e FC foram obtidas para avaliar a evolução hemodinâmica dos animais. As análises estatísticas destas variáveis foram realizadas nas seguintes fases dos diferentes grupos: sinais vitais iniciais (SVI), início da

Tabela 1 - Valores médios das variáveis pré-operatórias conforme o grupo estudado.

\begin{tabular}{|c|c|c|c|c|c|}
\hline Grupo Variável & Controle & Choque & Pringle & Total & $\mathbf{P} *$ \\
\hline $\boldsymbol{P A \boldsymbol { M }}(\mathrm{mmHg})$ & $118,75 \pm 17,91$ & $126,75 \pm 17,46$ & $136,50 \pm 14,88$ & $128,25 \pm 19,16$ & 0,265 \\
\hline $\boldsymbol{F} \boldsymbol{R}(\mathrm{mov} / \mathrm{min})$. & $60,25 \pm 8,58$ & $58,00 \pm 4,78$ & $63,50 \pm 16,48$ & $63,50 \pm 6,90$ & 0,648 \\
\hline $\boldsymbol{F C}(\mathrm{bpm})$ & $310,50 \pm 28,27$ & $292,50 \pm 40,02$ & $307,50 \pm 15,62$ & $312,00 \pm 19,24$ & 0,478 \\
\hline$P H$ & $7,329 \pm \quad 0,062$ & $7,257 \pm 0,059$ & $7,311 \pm 0,049$ & $7,295 \pm 0,033$ & 0,059 \\
\hline $\mathbf{P C O}_{2}(\mathrm{mmHg})$ & $49,15 \pm \quad 6,52$ & $57,67 \pm 9,50$ & $48,48 \pm 3,00$ & $52,66 \pm 5,45$ & 0,035 \\
\hline $\mathbf{P O}_{2}(\mathrm{mmHg})$ & $208,25 \pm 118,62$ & $240,37 \pm 68,62$ & $161,75 \pm 92,68$ & $222,00 \pm 99,11$ & 0,421 \\
\hline $\mathrm{HCO}_{3}^{-}(\mathrm{mmol} / \mathrm{l})$ & $27,25 \pm 3,98$ & $25,37 \pm 2,23$ & $24,81 \pm 1,75$ & $25,57 \pm 1,30$ & 0,269 \\
\hline $\boldsymbol{B} \boldsymbol{E}(\mathrm{mmol} / \mathrm{l})$ & $0,02 \pm 2,18$ & $-2,23 \pm 1,90$ & $-1,46 \pm 2,37$ & $-1,00 \pm 1,15$ & 0,161 \\
\hline $\mathbf{N a}^{+}(m E q / l)$ & $136,00 \pm 8,01$ & $132,14 \pm 4,14$ & $129,37 \pm 8,95$ & $133,62 \pm 10,33$ & 0,444 \\
\hline $\boldsymbol{K}^{+}(m E q / l)$ & $4,92 \pm 0,59$ & $4,30 \pm 0,74$ & $4,93 \pm 0,58$ & $4,60 \pm 0,72$ & 0,201 \\
\hline Lactato $(\mathrm{mmol} / \mathrm{l})$ & $0,92 \pm \quad 0,12$ & $0,95 \pm 0,19$ & $0,91 \pm 0,20$ & $0,85 \pm 0,14$ & 0,678 \\
\hline
\end{tabular}

*Análise de Variância (ANOVA).

reperfusão (RP0), reperfusão 30 minutos (RP30) e reperfusão 60 minutos (RP60). O gráfico 1 ilustra, mais detalhadamente, a média dos valores da PAM nos diferentes grupos, ao longo de todo o experimento.

Na comparação entre os valores da PAM no início do experimento (SVI), não foi verificada qualquer diferença significativa entre os diferentes grupos $(p=0,265)$, estando todos equiparados quanto a esta variável hemodinâmica. Na fase inicial da reperfusão (RP0), todos os grupos apresentavam PAM significativamente diferentes $(p<0,0001)$, exceto entre os grupos Pringle e Total $(\mathrm{p}=0,123)$, que evoluíram com valores muito próximos $(43,25 \pm 3,01 \mathrm{mmHg}$ e $46,00 \pm 3,46 \mathrm{mmHg}$ ) e bem abaixo dos demais grupos. Aos 30 minutos de reperfusão (RP30) apenas o grupo Controle apresentava diferença significativa com os demais grupos, com PAM apresentando valor superior a estes $(96,5 \pm 14,8 \mathrm{mmHg})$. Após 60 minutos de reperfusão (RP60) a medida da PAM mostrou-se novamente diferente apenas no grupo Controle que, como já vimos, apresentou valores acima dos demais grupos durante todo o experimento, exceto nos sinais vitais iniciais (SVI).
O número de neutrófilos por campo, presente no fígado, em cada grupo, após 60 minutos de reperfusão, fulcro do presente trabalho, está representado no gráfico 2 .

Gráfico 1 - Valores da PAM em cada fase do experimento conforme o grupo estudado.

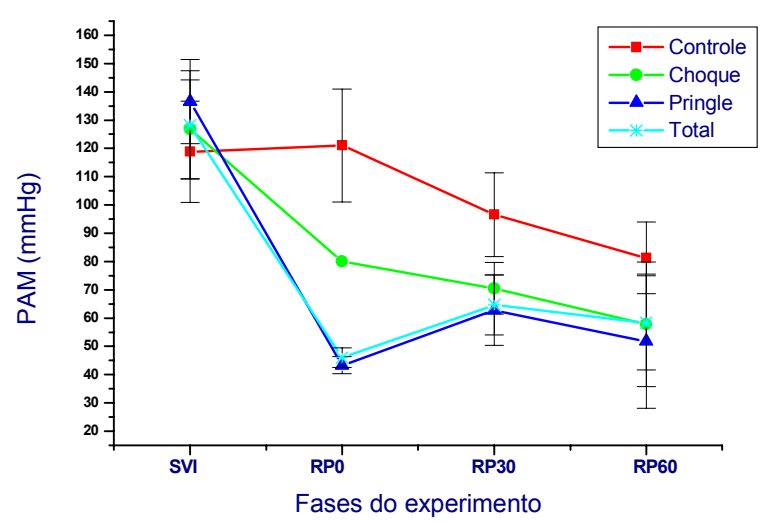

SVI - Sinais vitais iniciais RPO - Reperfusão 0 min. RP30Reperfusão $30 \mathrm{~min}$. RP60-Reperfusão $60 \mathrm{~min}$. 
Gráfico 2 - Valores do acúmulo de neutrófilos, por campo, no figado conforme o grupo estudado.

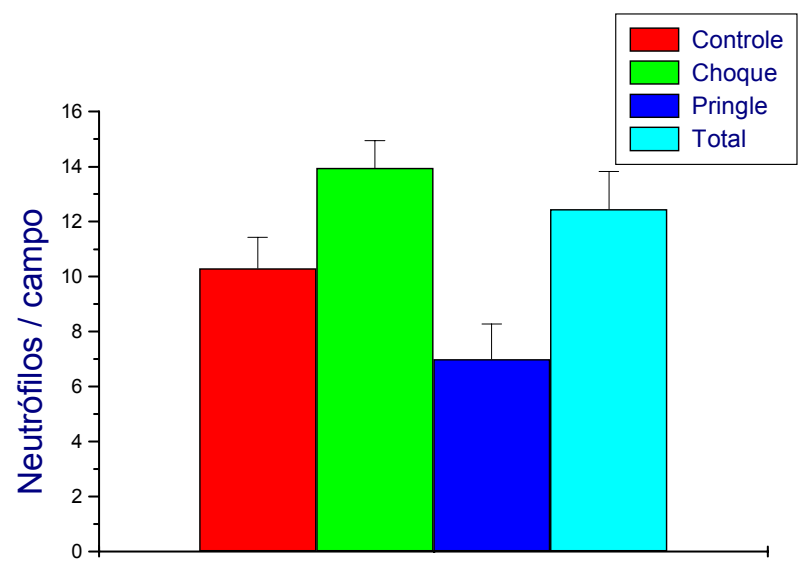

Grupos estudados

\section{DISCUSSÃO}

No trauma hepático as ressecções extensas continuam como raras indicações, sendo reservadas, apenas, quando não é possível outro tipo de procedimento e, nestes casos, coibir a hemorragia pelo clampeamento do pedículo hepático, durante o ato cirúrgico, é fundamental para o sucesso do tratamento ${ }^{8-10}$.

$\mathrm{Na}$ literatura, diversos autores preconizam que o tempo de isquemia hepática, se contínuo, não deverá exceder 30 minutos e, o procedimento do clampeamento intermitente deverá ser adotado quando o período para correção das lesões superar este valor. Porém, todos são unânimes em afirmar que a redução do tempo é fator importante na prevenção das lesões decorrentes da isquemia do órgão e, também, na prevenção de possíveis danos causados a órgãos distantes ${ }^{4,10}$.

Em geral, a exata etiologia destas alterações ainda demanda melhores esclarecimentos mas, freqüentemente, tem sido associada à isquemia própria do fígado, bem como às alterações da drenagem venosa com estase sangüínea do leito esplâncnico, a montante, que resultam em isquemia intestinal secundária à obstrução portal provocada. Embora o fígado possa suportar grandes danos sem que haja considerável comprometimento da função, este importante órgão da economia mostra-se bastante sensível à hipoxia e aos efeitos da reperfusão subseqüente que, de maneira associada, induzem à alterações, atribuídas, pelo menos em parte, à liberação de radicais li- vres durante a fase de reperfusão $0^{4,9,11,12}$. Deste modo, as lesões induzidas pela síndrome de $\mathrm{I} / \mathrm{R}$ podem ser atribuídas a dois mecanismos distintos. Um, caracterizado pela produção excessiva e posterior liberação de radicais oxigênio, altamente reativos e citotóxicos, durante a fase de reperfusão; o outro, pela interação dos polimorfonucleares neutrófilos (PMN) com as células endoteliais capilares, mediada pelas citocinas inflamatórias e moléculas de adesão ${ }^{13-16}$. Assim, procedimentos que causam a I/R hepática total, ou mesmo parcial, induzem à síntese destas citocinas que podem estar diretamente relacionadas ao desenvolvimento da resposta inflamatória sistêmica (SIRS) ou mesmo pela insuficiência de múltiplos órgãos e sistemas (IMOS) $)^{17-19}$.

Colletti et al. ${ }^{18}$ utilizando experimentalmente o procedimento descrito por Pringle, em ratos, procuraram determinar os efeitos da I/R hepática na liberação de mediadores químicos da resposta inflamatória, com ênfase para o TNF-a, concluindo ser esta substância o principal mediador envolvido nas alterações ocorridas no fígado e pulmão. Por outro lado, Gonce et al. ${ }^{4}$ com procedimento semelhante, avaliando a importância do tempo de I/R na indução do estado de choque circulatório, relataram que estas alterações se devem mais à isquemia intestinal induzida pela oclusão da tríade portal do que à própria hipoxemia e reperfusão hepática. Já, Bailey ${ }^{20}$ utilizando a dosagem de enzimas hepáticas para avaliar os danos que se seguem após 90 minutos de $\mathrm{I} / \mathrm{R}$ do fígado, afirmaram que, neste órgão, não ocorreram alterações importantes sobre as principais funções metabólicas, embora, tenha havido altos valores enzimáticos no plasma e morte celular acinar.

Os polimorfonucleares neutrófilos são considerados de grande importância na gênese das alterações fisiopatológicas que ocorrem após a liberação de mediadores em resposta à $\mathrm{I} / \mathrm{R}$. Neutrófilos seqüestrados nos locais da inflamação são ativados e liberam radicais superóxidos, além de enzimas proteolíticas que levam à lesão endotelial, aumento de permeabilidade microvascular, edema e lesão tecidual ${ }^{21}$. Portanto, a I/ $\mathrm{R}$ além de levar à lesão do próprio fígado por ação direta sobre os hepatócitos, também aumenta o número de polimorfonucleares neutrófilos e a permeabilidade microvascular nos tecidos de outros órgãos da economia, como no pulmão, sugerindo que a lesão hepática pode ser um fator desencadeante que contribui para o início da SIRS e, consequentemente da IMOS, em pacientes vítimas de trauma hepático extenso, princi- 
palmente, quando associado com grave estado de choque hemorrágico ${ }^{18,22}$.

Neste sentido, a contagem de PMN encontrados nos tecidos de diversos órgãos submetidos ao processo de I/R vem sendo utilizada como importante marcador para quantificar o grau de lesão associada e, neste trabalho experimental, tentou-se estabelecer uma correlação entre o acúmulo de neutrófilos no tecido hepático e a ocorrência de duas condições clínicas freqüentemente associadas - o choque hemorrágico e isquemia hepática.

Com os resultados obtidos, verificou-se que, na análise de comparação múltipla entre os diferentes grupos estudados, ocorreu uma diferença estatisticamente significativa $(\mathrm{p}=0.002)$ quanto ao acúmulo de neutrófilos no tecido hepático após um período de 60 minutos de reperfusão, com predomínio nos animais submetidos ao estado de choque hemorrágico (grupos Choque e Total) os quais, diferente do que se poderia esperar, apresentaram valores muito próximos e pouco diferiram dos animais do grupo Controle. Estes valores sugerem que o estado de choque hemorrágico, quando presente, por um período de 20 minutos, associado ou não à manobra de Pringle, não foi fator fundamental para o acúmulo dessas células no tecido hepático destes animais, sendo os resultados confirmados pela análise estatística de comparação simples (grupos dois a dois), que não mostrou diferença significativa entre os grupos aos quais o estado de choque hemorrágico foi realizado quando comparados com os animais do grupo Controle.
Por outro lado, sabe-se que, seja qual for a origem da isquemia, como em um órgão isolado, tal qual ocorre no pinçamento do pedículo hepático, ou mesmo generalizada, como no choque hemorrágico, sua instalação está relacionada ao posterior desencadeamento da resposta inflamatória, traduzida pelo 'recrutamento' e ativação de neutrófilos no sítio da lesão ou, de maneira sistêmica, com repercussão em órgãos distantes.

Nesta pesquisa, verificou-se que os animais do grupo Pringle apresentaram os menores valores na contagem de neutrófilos quando comparados aos demais grupos, uma vez que, nos animais onde foi empregada isoladamente ocorreram diferenças significativas quando comparados com os animais do grupo Choque $(\mathrm{p}<0.001)$ e Total $(\mathrm{p}=0.014)$, porém sem diferir do grupo Controle $(\mathrm{p}=0.075)$, representando, portanto, a manobra de Pringle, quando não associada ao estado de choque hemorrágico, um fator determinante para diminuir ou retardar o acúmulo de neutrófilos no tecido hepático.

Neste estudo experimental pode-se concluir que, em ratos submetidos a estado de choque hemorrágico controlado associado à isquemia hepática total por 15 minutos, seguido de 60 minutos de reperfusão, não ocorreu acúmulo significativo de neutrófilos no fígado. Este fato valoriza a técnica de tratamento das lesões hepáticas pelo clampeamento contínuo do pedículo hepático, mesmo na presença de grave estado de choque hemorrágico.

\begin{abstract}
Background: The purpose of this experimental study was to evaluate the effects of total hepatic ischemia and reperfusion on the accumulation of neutrophils in the liver of rats, under normal conditions and in rats submitted to controlled hemorrhagic shock. Methods: Thirty two adult male Wistar rats, were divided into four groups: the Control group, was submitted to the standard procedures for a period of 60 min of observation; Shock group, was submitted to controlled hemorrhagic shock (mean arterial blood pressure $=40 \mathrm{mmHg}, 20 \mathrm{~min}$ ) followed by volume resuscitation (lactated Ringer's solution + blood, 3:1) and reperfusion for $60 \mathrm{~min}$; Pringle group, was submitted to total hepatic ischemia for 15 min and reperfusion for $60 \mathrm{~min}$; The Total group, was submitted to controlled hemorrhagic shock for 15 min followed by volume resuscitation (lactated Ringer's solution + blood, 3:1), total hepatic ischemia for 15 min and reperfusion for $60 \mathrm{~min}$. Measurements of serum lactate and base excess were used to characterize the hemorrhagic shock state with low tissue perfusion. The counting of neutrophils on the liver tissue was performed after the euthanasia of animals. Results: Values for the counting of neutrophils on the liver indicate that, the animals from Pringle group differed from Shock and Total groups (Control 10.30 \pm 3.20 , Shock 13.94 \pm 2.84 ,

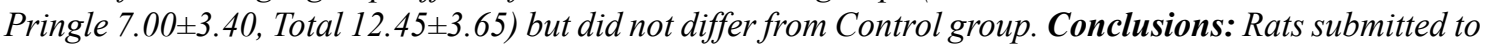
controlled hemorrhagic shock state associated to total hepatic ischemia for 15 minutes, followed by 60 minutes of reperfusion, did not present significant neutrophils accumulation on liver tissue.
\end{abstract}

Key Words: Ischemia; Reperfusion; Shock, hemorrhagic; Liver; Neutrophil; Rats, wistar; Hypovolemia. 


\section{REFERÊNCIAS}

1. Cirocchi R, Contine A, Mazieri M, et al. - Il packing periepatico associato a wrapping nel trattamento dei grandi traumi epatici bilobari. Chir Ital, 1999, 51(3):259-264.

2. Saccia A - La resezione maggiore nel trauma epatico grave. Indicazioni tecniche e considerazioni cliniche su di un caso di personale esperienza. Minerva Chir, 1994, 49(7-8):705-715.

3. Smith N-Pringle's maneuver. Curr Surg, 1982, 39(1):12-17.

4. Gonce ME, Brackett DJ, Squires RA, et al. Development of circulatory and metabolic shock following transient portal triad occlusion. J Surg Res, 1995, 59(5):534-543.

5. Uchinami M, Muraoka R, Horiuchi T, et al. - Effect of intermittent hepatic pedicle clamping on free radical generation in the rat liver. Surgery, 1998, 124(1):49-56.

6. Man K, Fan ST, Ng IO, et al. - Tolerance of the liver to intermittent pringle maneuver in hepatectomy for liver tumors. Arch Surg, 1999, 134(5):533 -539.

7. Sakamoto Y, Makuuchi M, Takayama T, et al. - Pringle's maneuver lasting $322 \mathrm{~min}$. Hepatogastroenterology, 1999, 46(25):457-458.

8. Towfigh S, Heisler T, Rigberg DA, et al. - Intestinal ischemia and the gut-liver axis: an in vitro model. J Surg Res, 2000, 88(2):160-164.

9. Man K, Fan ST, Ng IO, et al. - Prospective evaluation of Pringle maneuver in hepatectomy for liver tumors by a randomized study. Ann Surg, 1997, 226(6):704 - 713.

10. Isozaki H, Adam R, Gigou M, et al. - Experimental study of the protective effect of intermittent hepatic pedicle clamping in the rat. Br J Surg, 1992, 79(4):310-313.

11. Turnage RH, Kadesky KM, Myers SI, et al. - Hepatic hypoperfusion after intestinal reperfusion. Surgery, 1996, 119(2):151-160.

12. Kohli V, Selzner M, Madden JF, et al. - Endothelial cell and hepatocyte deaths occurs by apoptosis after ischemia-reperfusion injury in the rat liver. Transplantation, 1999, 67(8):1099-1105.
13. Evora PRB, Pearson PJ, Seccombe JF, et al. - Caracterização da lesão de isquemia/reperfusão. In Silva Jr OC, Zucoloto S, Beer Jr A (eds) - Modelos experimentais de pesquisa em cirurgia. São Paulo. Robe Editoral, 1998, pp. $433-438$.

14. Grinyo JM - Reperfusion injury. Transplant Proc, 1997, 29(1-2):59-62.

15. Dorinsky PM, Gadek JE - Mechanisms of a multiple nonpulmonary organ failure in ARDS. Chest, 1989, 96(4):885-892.

16. Christman JW, Holden EP, Blackwell TS - Strategies for blocking the systemic effects of cytokines in the sepsis syndrome. Crit Care Med, 1995, 23(5):955-963.

17. Schmeling DJ, Caty MG, Oldham KT, et al. - Evidence for neutrophil-related acute lung injury after intestinal ischemia-reperfusion. Surgery, 1989, 106(2):195-202.

18. Colletti LM, Remick DG, Burtch GD, et al. - Role of tumor necrosis factor-a in the pathophysiologic alterations after hepatic ischemia/reperfusion injury in the rat. J Clin Invest, 1990, 85(6):1936 - 1943.

19. Wanner GA, Ertel W, Müller P, et al. - Liver ischemia and reperfusion induces a systemic inflammatory response through Kupffer cell activation. Shock, 1996, 5(1):34-40.

20. Bailey SM, Reinke LA - Effect of low flow ischemiareperfusion injury on liver function. Life Sci, 2000, 66(11):1033-1044.

21. Weiss SJ - Tissue destruction by neutrophils. N Engl J Med, 1989, 320(6):365-376.

22. Calhoon JH, Trinkle JK. Pathophysiology of chest trauma. Chest Surg Clin N Am, 1997, 7(2):199-211.

Endereço para correspondência:

Mauro Fontelles

Rua Antônio Barreto, 983/1502

CEP 66055-050 - Belém - Pará

Fone :(091)225-1850/241-8601

E-mail:mikefox@uol.com.br 